\title{
Tangence
}

\section{Féminisme, postmodernisme et texte-supplément}

\section{Barbara Havercroft}

Numéro 39, mars 1993

La fiction postmoderne

URI : https://id.erudit.org/iderudit/025752ar

DOI : https://doi.org/10.7202/025752ar

Aller au sommaire du numéro

Éditeur(s)

Tangence

ISSN

0226-9554 (imprimé)

1710-0305 (numérique)

Découvrir la revue

Citer cet article

Havercroft, B. (1993). Féminisme, postmodernisme et texte-supplément.

Tangence, (39), 51-61. https://doi.org/10.7202/025752ar d'utilisation que vous pouvez consulter en ligne.

https://apropos.erudit.org/fr/usagers/politique-dutilisation/ 


\section{Féminisme, postmodernisme et texte-supplément}

\section{Barbara Havercroft}

Il y a, après tout, une différence entre vouloir véritablement penser différemment et penser le Même en manipulant la différence.

Alice Jardine, Gynésis: Configurations de la femme et de la modernité

Si les premiers écrits théoriques gravitant autour du postmodernisme ${ }^{1}$ sont caractérisés par leur profusion, leur hétérogénéité ainsi que par la contradiction ${ }^{2}$, ils le sont également par la signature presque exclusivement masculine de leurs auteurs ${ }^{3}$. Viennent

1 Il ne s’agira ici ni d'énumérer toutes les diverses conceptions du terme - postmoderne*, ni de décrire toutes les caractéristiques (telles que l'impureté, l'hybridité, le collage, etc.) des œuvres littéraires et artistiques postmodernes. Voir, entre autres, Ingeborg Hoesterey, "Introduction: Postmodernism as Discursive Event, dans I. Hoesterey (éd.), Zeitgeist in Babel: The Posimodernist Controversy, Bloomington, Indiana University Press, 1991, p. ix-xv; Linda Hutcheon, A Poetics of Postmodernism: History, Theory, Fiction, New York, Routledge, 1988; Guy Scarpetta, L'impureté, Paris, Grasset, 1985; Gianni Vattimo, La fin de la modernitê: nibilisme et berméneutique dans la culture postmoderne, Paris, Seuil, 1987; et les autres références dans la bibliographie du présent numéro de Tangence.

2 Comme la rubrique «postmoderne * constitue, selon Eco, *un terme bon à tout fairex, il vaut peut-être mieux le concevoir en tant que "catégorie spirituelle... une façon d'opérer. Voir Umberto Eco, Apostille au Nom de la rose, Paris, Grasset, 1985 , p. 74-75. Ou bien, comme le suggère Suleiman, on peut déplacer le débat postmoderne pour le situer en termes de ce qu'il fait (à l'intérieur d'un contexte spécifique), au lieu de le penser en termes de ce qu'il est. Voir Susan Suleiman, *Feminism and Postmodernism: A Question of Politics *, dans I. Hoesterey, op. cit., p. 119.

3 Dans un texte qui s'est révélé par la suite avoir joué le rôle d'un vrai catalyseur au sein de la polémique féminisme / postmodernisme, Owens affirme que son but était d'introduire la question de la différence sexuelle dans le débat "modernisme/postmodernisme", tout en soulignant l'indifférence «scandaleuse * de ce débat envers les théories féministes. Voir Craig Owens, "The Discourse of Others: Feminism and 
ensuite se greffer à ce corpus surtout masculin de théories et pratiques postmodernes un "corps " autre, tout aussi hétérogène, d'écrits et d'œuvres artistiques féminins. Ce supplément à la fois nécessaire et dangereux, pour reprendre les termes de Derrida ${ }^{4}$, a véritablement bouleversé les discussions autour du postmoderne, pour que "l'interpellation réciproque "s entre féminisme et postmodernisme devienne une problématique à part, qui fait actuellement l'objet de nombreuses études ${ }^{6}$. Autrement dit, ce qui était occulté dans les marges des marges s'est déplacé pour se rapprocher du centre du débat postmoderne.

Comme plusieurs critiques l'ont déjà constaté, les théories et pratiques féministes et postmodernes partagent un certain nombre de prémisses: elles présentent, par exemple, une critique des grands récits (de Dieu, de la Raison, de l'Homme, etc.) et des idéaux datant du siècle des Lumières sur lesquels se base notre culture $^{7}$; elles proposent des critiques de la représentation et des

Postmodernism, dans Hal Foster (éd.), The Anti-Aestbetic: Essays in Postmodern Culture, Seattle, Bay Press, 1983, p. 59.

4 Comme Derrida l'affirme, * le concept de supplément [...] abrite en lui deux significations dont la cohabitation est aussi étrange que nécessaire. Le supplément s'ajoute, il est un surplus [...] en tant que substitut il ne s'ajoute pas simplement à la positivité d'une présence, [...] sa place est assignée dans la structure par la marque d'un vide. Voir Jacques Derrida, De la grammatologie, Paris, Minuit, 1967, p. 208.

5 Nous reprenons ici l'expression dont Yeatman se sert pour décrire l'alliance précaire du féminisme et du postmodernisme. À cet égard, voir Anna Yeatman, The Epistemological Politics of Postmodern Feminist Theorizing *, Social Semiotics: A Transdisciplinary Journal in Functional Linguistics, Semiotics, and Critical Theory, vol. I, $\mathrm{n}^{\circ} 1,1991$, p. 30-48.

6 C'est surtout dans les années quatre-vingt que l'apport des femmes aux théories et pratiques postmodernes devient évident. D'après Suleiman, au cours des années soixante-dix et quatre-vingt, la production par des femmes d'œuvres littéraires et visuelles expérimentales a atteint un tel point *en termes de quantité et de qualité que l'on ne pouvait plus ignorer cette production artistique féminine. Voir Susan Suleiman, op. cit., p. 114. Puisque les études consacrées aux rapports entre féminisme et postmodernisme sont nettement trop nombreuses pour les citer toutes dans le contexte de la présente étude, nous nous contenterons de signaler les titres suivants: Jane Flax, Thinking Fragments: Psychoanalysis, Feminism, and Postmodernism in the Contemporary West, Berkeley, University of California Press, 1990; E. Ann Kaplan, Postmodernism and Its Discontents, London, Verso, 1988; et Meaghan Morris, The Pirate's Fiancée, Feminism, Reading, Postmodernism, London, Verso, 1988.

7 Consulter, entre autres, l'article de Craig Owens, op. cit., et Linda Nicholson et Nancy Fraser, *Social Criticism without Philosophy: An 
oppositions binaires pour favoriser le brouillage des frontières traditionnelles qui sous-tendent ces oppositions (telles celles entre le féminin/le masculin, l'art/la vie, le dominant/le marginal) ${ }^{8}$; enfin, elles tentent de combler la brèche entre théorie et pratique $^{9}$ et d'engendrer une multitude de petits récits hétérogènes, dont aucun ne prétend à l'autorité des grands récits occidentaux ${ }^{10}$. Situées simultanément à l'intérieur et à l'extérieur des idéologies dominantes, les théories et pratiques féministes et postmodernes se servent de la représentation pour contester ces idéologies et pour donner des voix, voire des voies, à de nouvelles formes et «normes" plurielles ${ }^{11}$. La rencontre entre féminisme et postmodernisme est donc susceptible d'apporter aux théories et pratiques postmodernes, d'une part, la visée et la force politiques qu'il leur manque, et d'autre part, une mise en cause radicale de la fiction humaniste de l'homme occidental en tant que sujet universel. Il importe de noter que la déconstruction de la fiction susmentionnée est partie intégrante d'un féminisme qui tient à démystifier une conception métaphysique et essentialiste de la Femme et à la remplacer par une catégorie plurielle et hétérogène des femmes ${ }^{12}$. Au lieu d'être figée et essentialiste, l'identité est à concevoir en fonction de son contexte, du gender, et à partir des systèmes de pouvoir et de signification particuliers qui la structurent ${ }^{13}$.

Encounter between Feminism and Postmodernism ", dans L. Nicholson (éd.), Feminism/Postmodernism, New York, Routledge, 1990, p. 19-38.

8 Voir Patricia Waugh, Feminine Fictions: Revisiting the Postmodern, New York, Routledge, 1990, p. 6.

9 Comme l'explique Barbara Creed dans son article « From Here to Modernity: Feminism and Postmodernism ", Screen, vol. XXVIII, $\mathrm{n}^{\circ} 2$, 1987, p. 48.

10 Pour une discussion de la problématique des grands et petits récits dans le contexte postmoderne, voir les écrits bien connus de Jean-François Lyotard, La condition postmoderne, Paris, Minuit, 1979, et Le postmoderne expliqué aux enfants, Paris, Galilée, 1988.

11 Linda Hutcheon, The Politics of Postmodernism, New York, Routledge, 1989, p. 23.

12 Sur la différence entre la Femme et les fermmes dans le contexte de ce débat, voir Sally Robinson, Engendering the Subject: Gender and SelfRepresentation in Contemporary Women's Fiction, Albany, State University of New York Press, 1991, p. 1-12.

13 Malgré ce portrait rassurant de la rencontre entre postmodernisme et féminisme, il existe des dangers importants. Non seulement le postmodernisme risque-t-il de retirer du féminisme sa force politique (par la voie de la détotalisation), mais on pourrait également se demander si les 
54

Le statut du féminisme comme supplément nécessaire au postmodernisme se reflète dans un conte de l'auteure allemande Christa Wolf intitulé Auto-expérimentation: rapport annexe, un texte qui est lui-même relégué, de par son titre même, aux marges supplémentaires ${ }^{14}$. Site textuel de la critique de certains grands récits familiers, à savoir l'opposition binaire entre le féminin et le masculin, les idéaux de l'objectivité scientifique et de la Raison, les principes du marxisme, de la technologie et du patriarcat, Auto-expérimentation explore en outre les problèmes de l'identité. Or, le sujet transsexuel de ce récit se trouve être le lieu fluide de la subversion des stéréotypes de gender, tout en s'entremêlant sans cesse à une critique des grands récits susmentionnés. Il s'agira dans la brève analyse qui suit de montrer comment la notion derridienne de supplément, inextricablement liée au sexe supplémentaire dans ce texte, est elle-même supplémentée par le sujet double, un sujet entre des genres fixes et stables.

Lieu par excellence du processus de la gynésis telle que Jardine la conçoit ${ }^{15}$, le conte de Wolf a êté publié d'abord dans une revue allemande, Sinn und Form, en 1973. Trois ans plus tard, le conte a connu une existence supplémentaire, ayant été republié dans une anthologie allemande qui s'intitule Blitz aus beiterem Himmel (Un coup de tonnerre dans un ciel blew), recueil où tous les récits portent sur le changement de sexe. Il n'est pas question, dans le texte de Wolf, d'un androgyne postmoderne,

femmes sont en mesure d'épouser une conception de la pluralité décentrée, étant donné qu'elles ne se sont jamais définies comme sujet stables. Ces questions font l'objet d'analyses approfondies par Christine Di Stefano, *Dilemmas of Difference: Feminism, Modernity, and Postmodernism *, dans L. Nicholson (éd.), op. cit., p. 63-82 et également par L. Nicholson et N. Fraser, op. cit., p. 19-38.

14 Ceci en fonction de son statut textuel déclaré (un rapport annexe) et également en tant que texte européen postmoderne. Les ensembles les plus visibles et cohérents de la fiction postmoderne sont constitués de textes américains (des auteurs tels que Barth, Barthelme, Coover, etc.) ou de textes québécois (Aquin, Ducharme, Brossard, etc.).

15 Jardine définit la gynésis de la façon suivante: * la mise en discours de "la femme" comme processus [...] ou encore la valorisation du féminin, de la femme et de ses connotations obligatoires, c'est-à-dire historiques, comme faisant partie intrinsèque de nouveaux modes nécessaires de penser, d'écrire et de parler». Voir Alice Jardine, Gynésis: Configurations de la ferme et de la modernité, Paris, Presses universitaires de France, [1985] 1991, p. 24. 
mais d'un(e) transsexuel(le) qui combine les expériences "masculines" et "féminines" dans un seul et même corps. L'histoire se déroule en 1992, quand une femme scientifique se transforme en homme en prenant une drogue (Petersein Masculinum, p. 199), "un produit remarquable" ${ }^{6}$, selon la narratrice. Quoique cette expérience soit un succès biologique - la drogue transforme effectivement la femme en homme - les autorités scientifiques concluent qu'elle est un échec. La femme scientifique, sujet de l'expérience devenant de plus en plus son objet, demande le renversement de ce changement avant que la durée prévue de trois mois soit terminée.

Le rapport annexe et subjectif de cette narratrice anonyme explique sa décision de subir une telle transformation et ensuite, les raisons pour lesquelles elle a choisi de la renverser. Comme elle l'affirme dans ce rapport dialogique, elle voulait démontrer sa valeur en tant que femme "en acceptant de devenir un homme" (p. 91). Amoureuse du Professeur-directeur de l'Institut scientifique, elle emprunte, lors de ce devenir-homme, un corpssupplément, son corps à lui, en sacrifiant le sien, afin de donner corps à sa vision scientifique à lui et à ses besoins sentimentaux à elle. Double raisonnement qui s'achève dans un être double. Ainsi la femme écrit-elle le rapport annexe, suppléant de cette façon le rapport officiel de l'expérience, pour dévoiler le secret du "masculin". Révélant ce secret dans son rapport, elle en révèle également d'autres, puisqu'elle rompt avec l'exigence officielle de camoufler certains détails de cette recherche scientifique, le secret étant un des principes de base du grand récit scientifique de l'Allemagne de l'Est.

Par son portrait du sexe supplémentaire et de l'entre-genre, ce texte indique non seulement l'instabilité du corps, mais aussi l'engendrement arbitraire de l'expérience humaine à partir des rôles stéréotypés et appris, de même que l'inscription discursive de cette expérience dans un langage qui produit son sens à partir de la différence. La problématique de l'entre-genre entraîne une double logique paradoxale. Il s'agit d'un sujet qui n'est ni l'un ni l'autre; toutefois ce sujet est simultanément l'un et l'autre. C'est

16 Christa Wolf, *Auto-expérimentation: rapport annexe*, dans C. Wolf, Trois bistoires invraisemblables, Aix-en-Provence, Editions Alinéa, 1987, p. 82 . Toute référence au texte sera désormais indiquée par le numéro de page entre parenthèses. 
aux niveaux onomastique et pronominal que ce sujet équivoque et oscillant trouve l'expression de son identité flottante. Identifiée uniquement en tant que $j e$, signe "vide " et dépourvu de marque générique spécifique, la narratrice ne possède de nom propre qu'après la transformation - après avoir emprunté un corps d'homme. C'est alors que le professeur, jouant le rôle du Père doté du pouvoir de nomination, lui donne son nom: Anders (Autre). Ce renversement de genre, où le je devient un autre, voire un être double, implique un autre renversement, celui de la catégorie de "l'autre". La théorie féministe a tendance à concevoir la femme en tant que "l'Autre", catégorie "aussi originelle que la conscience elle-même" ${ }^{17}$. Appartenant d'abord à cette catégorie féminine de "l'autre" tout en s'en éloignant par sa profession et par son rôle traditionnellement masculins, la femme ne se désigne par ce titre qu'après être devenue autre, d'où un Autre masculin. Quant à la forme linguistique de ce nom propre, elle est aussi autre. L'emprunt du corps autre va de pair avec l'em-. prunt d'un adverbe (anders) qui fonctionnera dorénavant à la place du nom propre - il n'y avait aucun autre nom propre à nommer ce sujet autre. En outre, l'adverbe anders en allemand dénote "autrement" ou "de manière différente", et se combine avec d'autres formes lexicales pour produire des expressions composées telles que andersdenkend (d'une opinion différente; celui qui pense autrement) et andersgeartet (hétérogène).

Anders (Autre), nom significatif, souligne l'altérité de ce sujet double de plusieurs façons différentes. Anders est devenue autre à elle-même; toutefois, elle reste toujours "autre" aux hommes. En éliminant la frontière biologique rigide entre homme et femme, elle constitue littéralement un/une autre vis-à-vis de la différence sexuelle qui instaure et marque l'altérité. Une fois son corps devenu autre, son esprit demeure toujours "féminin" (au moins, au début du texte); ainsi est-il "autre" vis-à-vis du corps qui le contient. Au fur et à mesure que le temps se déroule, elle constate que même son esprit commence à devenir autre (masculin) pour ressembler de plus en plus à son corps - d'où sa décision de d'arrêter l'expërience. Au moment où l'altérité se définit uniquement par le masculin, Autre opte pour le "redevenir autre", le retour à la catégorie propre à l'autre - le féminin.

17 Simone de Beauvoir, Le deuxième sexe, tome I, Paris, Gallimard [1949] 1976, p. 16. 
Néanmoins, elle restera toujours *autre "à cause de cette expérience (ce mot est ici à comprendre dans ses deux sens), et par conséquent, elle ne sera jamais l'objet d'une altérité univoque.

Le processus progressif de devenir "autre" (c'est-à-dire l'inévitable acheminement vers la coïncidence du nom propre et de l'état du sujet), un apprentissage impliquant tous les clichés de la socialisation double (au masculin et au féminin), s'accompagne de la dissémination du nom propre "Autre "dans le texte. Autre apprend à penser autrement: elle/il pense à son propre sacrifice (elle avait renoncé à son amant et à son désir d'avoir un enfant pour poursuivre sa carrière). Autrefois, la pensée de ce sacrifice a évoqué un sentiment de regret, mais "au cours de ma première nuit d'homme, je pus pour la première fois $[\ldots$ y] penser sans remords... Ce que je ressentais, c'était de la gratitude " (p. 90). L'Andersdenken (le penser autrement) entraîne l'Andersfiublen (le ressentir autrement), l'Andersseben (le voir autrement), et même l'Andersessen (le manger autrement). Restent à suivre le parler- et le nommer autrement, qui se manifestent au niveau de la connotation d'un mot aussi simple que "la ville". Tandis que "la ville" connotait autrefois pour elle "une foule d'espoirs toujours déçus et toujours renaissants" (p. 98), ce mot évoque maintenant d'autres connotations: "une inépuisable concentration d'occasions" (p. 98). "Étourdi par une ville [qui] voulait [lui] enseigner que [s]on devoir était de faire des conquêtes" (p. 98), Autre a néanmoins accès à la fois aux deux connotations (l'ancienne et l'actuelle), puisque "la femme en moi n'avait pas encore désappris la tactique qui consiste à se mettre en avant et à filer doux si la situation l'exige" (p. 98).

De cette manière, l'emprunt du corps, et, peu à peu, de l'esprit supplémentaires illustre clairement la relation de supplémentarité qui existe entre sexe et genre (gender). $\mathrm{Si}$, comme le note Scott, "le genre [...] est une catégorie sociale imposée à un corps sexué "18, l'emploi du lexème "ville" dans ce contexte signale la naturalisation insidieuse de l'apprentissage et de l'emploi de la langue. Loin de fonctionner en tant que lexème innocent qui exprime une expérience libre de contraintes génériques, le mot "ville" s'avère le "signifiant d'un espace géographique qui produit et est le produit des processus différents

18 Joan Scott, "Gender: A Useful Category of Historical Analysis *, American Historical Review, vol. XCI, 1986, p. 1056 [ma traduction]. 
déterminés par le genre [gender] "19, processus qui sont imposés au sujet en fonction du corps biologique. Ces différences génériques deviennent encore plus manifestes au cours d'un test supplémentaire, où les réponses que fournit Autre aux stimuli linguistiques représentent, de façon ironique, toute une série d'oppositions binaires qui varient selon le sexe biologique et le genre qui lui est propre: "[...] en fin de compte, à "rouge" je répondis "colère" et non "amour" comme d'habitude" (p. 93). Ainsi Autre, en répondant autrement qu'auparavant, se moque-t-elle / il à la fois des clichés appris et prescrits et du positivisme scientifique.

Sur le plan pronominal, le texte accentue la problématique de l'autoréférence chez le sujet écrivant. Le "je" ne sait plus comment se référer - est-ce que c'est un "je" dont le référent est une "elle" ou un "ill"? Autre sait que la première personne, en principe, peut désigner le moi, un masque, ou un rôle:

Savez-vous ce que signifie "personne"? Masque. Rôle. Personnalité réelle. Le langage, me semble-t-il en fin de compte, est certainement lié à l'un au moins de ces trois états. Je les avais tous perdus, ce qui ne pouvait signifier que le silence total. Impossible de prendre des notes sur soi si l'on n'est "personne". D'où la lacune de trois jours dans mon compte rendu (p. 107-108).

Autre devient donc progressivement et littéralement une nonpersonne, non pas uniquement dans le sens de Benveniste, mais dans le sens où il ne lui reste aucun moyen discursif adéquat d'autoréférence. Sans l'existence de cette autoréférence, de cette existence linguistique, il/elle cesse d'exister. Sans la possibilité de devenir un sujet d'énonciation, il/elle ne parviendra pas à s'inscrire dans l'histoire, pas même dans sa propre histoire.

Le processus de devenir autre, cette transformation graduelle d'un état équivoque de dédoublement en être univoque et masculin, se distingue par ses étapes différentes. La combinaison initiale des habitudes, des souvenirs et des pensées de femme, enfermés dans un corps d'homme, se manifeste d'abord par la confusion (la Verwirrung) ${ }^{20}$, où Autre sent son sourire de femme

19 Anne Herrmann, "The Transsexual as Anders in Christa Wolf's "SelfExperiment", Genders, vol. III, automne 1988, p. 48 [ma traduction].

20 La version originale allemande du texte à laquelle nous faisons référence se trouve dans un recueil de contes de femmes intitulé Frauen in der DDR: Zwanzig Erzäblungen, Lutz-W. Wolff (éd.), Munich, Deutscher Taschenbuch Verlag, 1976. 
toujours se former en lui/elle, sans que ce sourire puisse parvenir à la surface, à son visage. Cette expérience de l'entre-genre, région fluide de la Verwirrung, combinaison des deux sans pour autant être l'un ou l'autre, Autre la compare à une *zone floue du no man's land" où il ne ressent rien sauf "un léger début de nostalgie" (p. 95). Peu à peu, la partie "femme" (l'esprit autre) commence à disparaître, processus qui se caractérise par l'emploi croissant de mots comportant le préfixe ver-en allemand, un préfixe qui indique souvent, devant un verbe réflexif, une intensification, un changement, un devenir. Ainsi la Verwirrung cèdet-elle sa place au Verlust (mot qui signifie à la fois la perte, le deuil et les dommages), à la Verwandlung (la métamorphose), aux Verklemmungen (l'inhibition, le refoulement) et aux Verkrampfungen (la tension, le resserrement). La dissolution de l'opposition binaire entre le féminin et le masculin, cette mise en question de la différence, finit enfin par se transformer en indifférence, et c'est cette indifférence qui constitue le secret du masculin tel que l'apprend Autre.

Le statut textuel de ce rapport annexe, à savoir celui d'un textesupplément, va de pair avec la notion de sexe supplémentaire. $\mathrm{Si}$ un supplément est une addition, d'abord considérée comme superflue, à une entité déjà complète, ce supplément s'y ajoute pourtant pour compléter, pour combler un vide au sein d'une entité qui n'a jamais été tout à fait complète. Le rapport annexe, en tant qu'appendice, noue en lui ce double sens du supplément. Ce textesupplément est vraisemblablement à lire avec le rapport officiel scientifique de l'expérience ou à y ajouter. Cependant, ce supplément constitue non seulement le texte le plus révélateur sur l'expérience, ce qui renverse la hiérarchie traditionnelle entre le texte principal et son supplément, mais ce rapport annexe prend entièrement la place du rapport officiel, son prétendu hypotexte, qui n'existe qu'à partir de quelques références dans l'hypertexte. Le texte subjectif et supplémentaire, le texte féminin, devient la seule "Source" de renseignements concernant cette expérience, en écartant entièrement le corps autre, le texte masculin, objectif et attendu. Ce supplément dangereux s'avère nécessaire, puisque la "vérité " de l'expérience, c'est-à-dire les expériences personnelles de la femme/homme pendant cette expérience, ne se manifestent pas dans le rapport scientifique neutre. Le texte-supplément féminin est ainsi suscité par ce qui manque au rapport scientifique officiel, masculin et absent: "Combler une lacune en décrivant sa genèse: 
60

peut-on imaginer prétexte plus brillant pour vous soumettre ces informations?" (p. 81).

De par sa nature dialogique, mettant en jeu un dialogue imaginaire entre Autre et son autre (le Professeur), le textesupplément substitue la représentation des relations interpersonnelles à celle du modèle masculin de l'acquisition d'une vérité univoque et monolithique, écartant le prototype du grand récit scientifique. Très consciente de la nature subjective de son rapport et des dangers de sa rupture avec le positivisme du rapport scientifique standard, Autre remarque "l'ambiance impersonnelle qui s'imposait " (p. 87) à l'Institut et va jusqu'à "s'excuser" de sa transgression des normes objectives: "Excusezmoi, je ne suis plus objective" (p. 88). Ce texte féminin, tout en racontant l'expérience du sexe supplémentaire, est double dès le départ, fait comme il l'est de questions et de réponses anticipées de la part de la femme lors de son dialogue imaginaire avec le Professeur. Parsemé de marques de son énonciation, ce texte met en relief son propre devenir, son propre processus d'inscription et de transcription de la réversibilité du je et du $t u$. Par son statut de supplément, ainsi que par sa forme dialogique et double, le rapport annexe ne se limite pas à la simple description du devenir autre, mais il le reflète. L'emprunt du corps masculin et autre se manifeste par le je équivoque qui tente de changer de place avec son vous interlocuteur, le Professeur. L'ajout du sexe supplémentaire et les diverses étapes impliquées par la tentative de faire coïncider corps et esprit autres se traduisent dans le texte dialogique d'abord par l'oscillation des référents pronominaux, ensuite par le silence de la Verwirrung, et finalement par un je dont le référent se caractérise de plus en plus par sa "masculinité" apprise. Réduit à l'indifférence dans laquelle il ne perçoit plus nettement la nature arbitraire et codée de la socialisation et sa bifurcation en deux ensembles de stéréotypes, Autre constate qu'à l'instar de Tirésias, héros aveuglé de l'intertexte implicite dans le texte-supplément, il est lui-même devenu aveugle:

Non, Professeur; il n'y aura pas de déesse pour descendre crever les yeux du traître - à moins que vous ne considériez comme une déesse toute puissante l'habitude qui nous rend aveugles. L'aveuglement partiel que s'infligent presque tous les hommes commença également à s'emparer de moi: comment, sinon, jouir aujourd'hui sans partage de privilèges quelconques? Là où autrefois je me serais révoltée, je restais indifférente (p. 109-110). 
L'aveuglement et l'indifférence de cet être "supplémenté" l'amènent à demander le renversement de l'expérience. Et ce faisant, elle/il rejette l'ajout du sexe-supplément pour devenir, encore une fois, autre. Toutefois, ce "redevenir féminin" n'implique pas pour autant un retour à une catégorie primordiale et essentielle. Autre reste toujours autre, ayant appris que la différence et la bifurcation du genre (gender) qui en résultent ne sont pas les conséquences nécessaires du sexe biologique. Au contraire, c'est plutôt les multiples processus contraignants de penser, de parler, d'agir autrement qui instaurent cette opposition binaire de rôles typiques.

À la différence du sexe-supplément rejeté, le textesupplément, quant à lui, reste le lieu discursif et nécessaire de l'inscription de cette expérience. De cette façon, il revendique la possibilité féminine d'affirmer l'ex-centrique, l'hétérogène et la critique des catégories binaires immuables. Ainsi le texte de Christa Wolf participe-t-il pleinement de la pratique postmoderne qui offre des voix à l'autre, ou bien qui donne la parole à d'autres voix jadis opprimées ou supprimées. À sa mise en question d'un sujet univoque vient s'ajouter la voie de l'expérience multiple. Loin d'aboutir à une série infinie et insensée de différences multiples, à de simples jeux langagiers gratuits, une visée et une vision féministes sont susceptibles, comme en témoigne la pratique scripturale de Christa Wolf dans Auto-expérimentation, d'incarner, voire d'incorporer, la force politique qui manque au postmodernisme - bref, d'en constituer le supplément nécessaire. 